\title{
Online Detection of Freezing of Gait in Parkinson's Disease Patients: A Performance Characterization
}

\author{
Marc Bächlin \\ Wearable Computing Lab. \\ ETH Zürich ${ }^{1}$ \\ baechlin@ife.ee.ethz.ch \\ Jeffrey M. Hausdorff \\ Lab. for Gait and \\ Neurodynamcis, TASMC ${ }^{2}$ \\ jhausdor@tasmc.health.gov.il
}

\author{
Daniel Roggen \\ Wearable Computing Lab. \\ ETH Zürich ${ }^{1}$ \\ roggen@ife.ee.ethz.ch \\ Nir Giladi \\ Lab. for Gait and \\ Neurodynamcis, TASMC ${ }^{2,4}$ \\ nirg@tasmc.health.gov.il
}

\author{
Meir Plotnik \\ Lab. for Gait and \\ Neurodynamcis, TASMC ${ }^{2,3}$ \\ meirp@tasmc.health.gov.il \\ Gerhard Tröster \\ Wearable Computing Lab. \\ ETH Zürich ${ }^{1}$ \\ troester@ife.ee.ethz.ch
}

\begin{abstract}
Freezing of gait (FOG) is a common gait deficit in advanced Parkinson's disease (PD). It is often a cause of falls, interferes with daily activities and significantly impairs quality of life. PD patients can be assisted by auditory cueing. In daily life cueing should be automatic during gait freeze.

This paper describes our ambulatory research platform for context-aware online FOG detection and auditory cueing. The system analyzes frequency components of body motion to detect FOG and provides a metronome sound until the patient resumes walking. We characterize the sensitivity and specificity of the system as functions of: sensor placement and orientation, walking style and algorithm parameters.

We have performed a study with ten PD patients, which have worn our system performing several walking tasks. Over $8 \mathrm{~h}$ of data has been recorded and 237 FOG events have been identified by professional physiotherapists in a post-video analysis. The system detected the FOG events online with a sensitivity of $73.1 \%$ and a specificity of $81.6 \%$. We show
\end{abstract}

\author{
*Corresponding author \\ ${ }^{1}$ Wearable Computing Lab. \\ Swiss Federal Institute of Technology Zürich \\ 8092 Zürich, Switzerland \\ http://www.ife.ee.ethz.ch \\ ${ }^{2}$ Laboratory for Gait and Neurodynamcis \\ Tel Aviv Sourasky Medical Center \\ Tel Aviv, Israel \\ http://www.tasmc.org.il/e/ \\ ${ }^{3}$ Gonda Brain Research Center \\ Bar Ilan University, Ramat Gan, Israel \\ http://www1.biu.ac.il/indexE.php \\ ${ }^{4}$ Department of Neurology \\ Sackler School of Medicine, Tel Aviv University, Israel \\ http://medicine.tau.ac.il/english/
}

that the theoretical maximum performance of this algorithm with patient specific optimal parameter sets is $88.6 \%$ sensitivity and $92.8 \%$ specificity. By separating the patients into saccadic and smooth walker with separate feature sets a detection accuracy of $85.9 \%$ sensitivity and $90.9 \%$ specificity was measured.

The vertical axis of the sensor at the knee is the best sensor position and orientation for FOG detection. However the detection performance is relatively insensitive to the location and orientation, showing the robustness of the algorithm.

\section{INTRODUCTION}

Context awareness is an essential basis for providing the relevant feedback and assistance in various health-related applications such as rehabilitation or elderly assistance.

On body sensing, data processing and user feedback are generally favored as they are 'worn', thus available anytime and anywhere. We consider here a self-contained lifestyle assistant for Parkinson's disease patients with the freezing of gait syndrome, including sensors, online data processing, and user auditory assistance.

In this paper we present the online FOG detection performance analysis using our modular research platform that consists of wireless acceleration sensor nodes and a wearable computing system. In detail our paper presents the following contributions:

1. A detailed description and complexity analysis of the real-time FOG detection algorithm that we developed and optimized for online execution in wearable systems.

2. A detailed performance analysis of the algorithm in offline use to evaluate the limits of this algorithm.

3. Evaluation of performance at three different body positions and orientation.

4. A summary of the positive outcomes from this wearable assistant identified from the field study with the Parkinson's disease patients. 


\section{MEDICAL BACKGROUND}

Parkinson's disease (PD) is a common neurological disorder, caused by a progressive loss of dopaminergic and other sub-cortical neurons [5]. PD often leads to impaired motor skills, speech, and other functions [7]. Beside a flexed posture, tremor at rest, rigidity, akinesia (or bradykinesia) and postural instability, motor blocks are a common negative effect of PD. Most commonly the legs are affected during walking, which is generally referred to as freezing of gait (FOG).

FOG typically manifests as a sudden and transient inability to move. About $50 \%$ of PD patients experience freezing, $10 \%$ of patients with mild PD symptoms and $80 \%$ of those severely affected by PD. Freezing occurs more frequently in men than in women and less frequently in patients whose main symptom is tremor [9]. Freezing is associated with substantial social and clinical consequences for patients. In particular, it is a common cause of falls [4], interferes with daily activities, and significantly impairs quality of life [6]. Gait deficits and FOG are often resistant to pharmacologic treatment $[4,12]$. Therefore effective non-pharmacologic treatments need to be developed as an adjunct therapy to relieve symptoms and improve mobility. Literature shows that PD patients can take advantage of external cueing, such as auditory rhythmic sound, to help themselves out of the freezes $[8,13,14]$. While this has been investigated with manual triggered sound in laboratory settings, in daily life cueing should be automatic during FOG. We investigate how to automatically detect and react to FOG.

\section{WEARABLE FOG ASSISTANT 3.1 Hardware}

Figure 1 shows our system and the accelerometer sensors as worn by a patient. Sensors measuring 3D-acceleration are attached to the shank (just above the ankle), to the thigh (just above the knee) and to the lower back (on the belt) using an elasticized strap and Velcro. The acceleration sensors are $27 \times 47 \times 12 \mathrm{~mm}^{3}$ in size and weight less than 22 gram, including a rechargeable Li-ion battery [2].

The wearable computing system for the data recording and online processing is a research platform based on an Intel XScale family processor and a Linux operating system, designed for rapid prototyping. It offers processing power comparable to an ultra portable PC. The system offers by default USB and Bluetooth as extension interfaces, allowing connections to a diversity of physiological and non-physiological sensors [2]. Due to the general-purpose and energy-efficient processor the system allows running online algorithms while the power consumption is between 1.5 and 2 Watts. On the $3.7 \mathrm{~V}, 3.3 \mathrm{Ah}$ battery the system can run for more than $6 \mathrm{~h}$. The wearable computer is $132 \times 82 \times 30 \mathrm{~mm}^{3}$ in size and weights 231 grams. It is also attached to the belt, placed around the trunk.

The wearable computer detects all available sensors automatically and start the online processing. The acceleration data $(64 \mathrm{~Hz})$ are transmitted over a wireless Bluetooth link to the computing system for real-time identification of FOG. Earphones placed around the patient's neck and connected to the computing system produce a $1 \mathrm{~Hz}$ ticking sound whenever an FOG episode is identified and last until the patient resume walking.

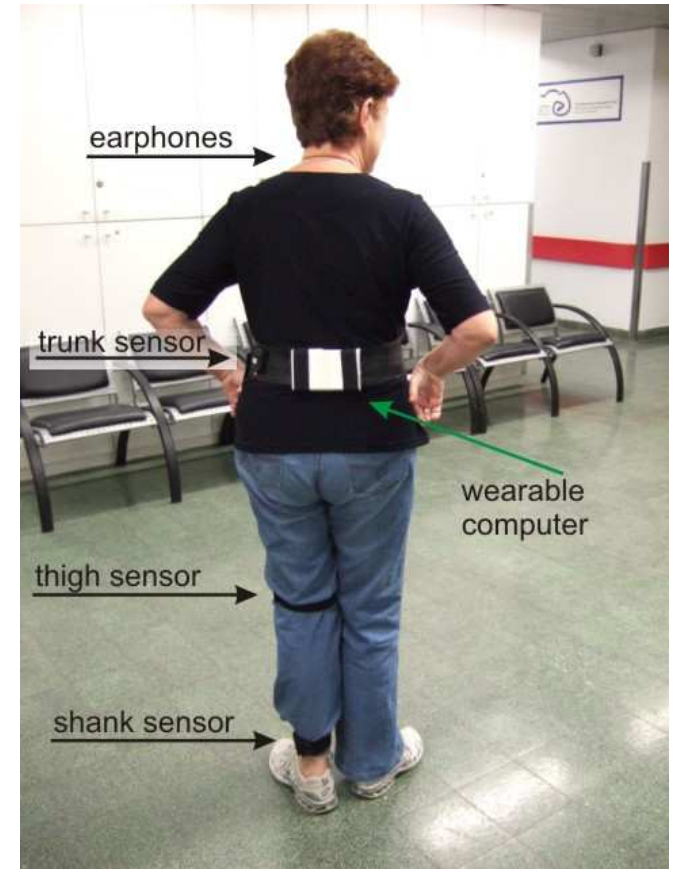

Figure 1: FOG detection and feedback device worn by one patient. Sensors are attached to the shank (just above the ankle) and the thigh (just above the knee) using an elasticized strap and Velcro. A third sensor is attached to the belt where also the wearable computer is attached to.

\subsection{Online FOG detection algorithm}

Moore et al. analyzed the power spectra of the vertical acceleration of the left shank on a $6 \mathrm{sec}$ window of $11 \mathrm{PD}$ patients [10]. They found that high-frequency components of leg movement during FOG in the $3-8 \mathrm{~Hz}$ band were not apparent during volitional standing or during walking. For an objective method to identify FOG automatically offline, Moore introduced a freeze index (FI), defined as the power in the 'freeze' band divided by the power in the 'locomotor' band $(0.5-3 \mathrm{~Hz})$. FOG can be detected using a 'freeze' threshold. FI values above this threshold are identified as FOG.

We developed an online FOG detection algorithm based on the principle described by Moore with emphasis on low latency. The latency of the algorithm is dominated by the window length needed for the fast fourier transformation (FFT) calculation. The smallest resolution of the FFT is determined by the sampling frequency $\left(f_{S R}\right)$ of the signal and the FFT-length $\left(N_{F F T}=\right.$ number of frequency points between ' $-\frac{f_{S R}}{2}$ ' and ' $+\frac{f_{S R}}{2}$ ') by the equation: $f_{\text {res }}=\frac{f_{S R}}{2}$. $\frac{1}{0.5 \cdot N_{F F T}}=\frac{f_{S R}}{N_{F F T}}$. Given a minimum $f_{\text {res }}$ the FFT must have at least $N_{F F T} \geq \frac{f_{S R}}{f_{r e s}}$ points. In order to be able to sum up the energy in the two frequency bands a minimum number of components is required. We decided to have at least 10 frequency components between $f_{0.5}=0.5 \mathrm{~Hz}$ and $f_{3}=3.0 \mathrm{~Hz}$. To achieve this $f_{\text {res }}$ has to be equal or smaller than $0.25 \mathrm{~Hz} /$ point $\left(f_{\text {res }} \leq \frac{f_{3}-f_{0.5}}{10 \text { points }}=\frac{2.5 \mathrm{~Hz}}{10 \text { points }}\right)$, and the 
window length must therefore be $N_{F F T} \geq \frac{f_{S R}}{f_{r e e}}=4 \mathrm{sec} \cdot f_{S R}$. In other words, the window length must be at least $4 \mathrm{sec}$ long, independent of the sampling frequency $\left(f_{S R}\right)$ used.

The energy in the 'Loco band', the low frequency part between 0.5 and $3 \mathrm{~Hz}$ is summed up as well as the energy in the 'Freeze band', the higher frequency part between 3 and $8 \mathrm{~Hz}$. We also compute the complete energy $\left(E_{\text {total }}\right)$ between $0.5 \mathrm{~Hz}$ and $8 \mathrm{~Hz}$ by the addition of both parts.

If the power content of the signal between $0.5-8 \mathrm{~Hz}$ is above the power-threshold (PowerTH), the freezing index (FI) is calculated by dividing the energy in the freeze band by the energy in the locomotor band. For signal parts with a power content $E_{\text {total }}$ below the PowerTH (standing parts), FI is set to zero. Finally FOG is detected whenever the FI exceeds the 'freeze' threshold (FreezeTH).

The PowerTH is needed to avoid that standing parts are detected as FOG. This can happen, because in these parts there is almost no energy in the two frequency band and a division of two very small numbers is very sensitive to small variations resulting in almost random output numbers. With the PowerTH the sensitivity of the algorithm for detecting volitional standing parts can be adjusted, respectively the specificity of the FOG detection by avoiding that volitional standing is detected as FOG.

The FreezeTH determines the sensitivity of the FOG detection. A too low FreezeTH introduces too many false detections. A too high FreezeTH results in missing events and increases the reaction time, because the FI must first rise up to the threshold value before FOG is detected (see figure 10).

The key differences to Moore's algorithm are a reduced, optimized window size, and the introduction of the second threshold. Both of these are necessary modifications to make it suitable for online use. A signal extract together with the FI and the parts where FOG is detected is depicted in figure 10 .

For the implementation on the wearable device we used the Context Recognition Network (CRN) Toolbox [3]. During the study only the shank sensor data has been used for the online FOG detection. Sensor data coming from the acceleration sensor with $64 \mathrm{~Hz}$ are windowed by a $4 \mathrm{sec}$ window. The windowing is done in steps of $0.5 \mathrm{sec}$.

\section{EVALUATION STUDY}

Ten idiopathic PD patients with a history of FOG, able to walk un-assisted in "OFF" period (at a non-effective level of medication) were recruited for our study by the specialists at the Movement Disorders Unit, at the Tel Aviv Sourasky Medical Center. The diversity of the participating patients covered a wide spectrum of PD patients (Hoehn-Yahr score (H\&Y) between 2-4). It included PD patients difficult to differentiate from healthy people by their gait performance when they do not have an FOG event, as well as a PD patient not able to walk distances $>15 \mathrm{~m}$ (often using a wheelchair in normal life). A detailed characteristic of the participants is given in [1].

The study protocol had two sessions, one without auditory feedback and one with auditory feedback. Each session consisted of 3 basic walking tasks, designed to represent normal daily walking. The first two tasks included straight walking

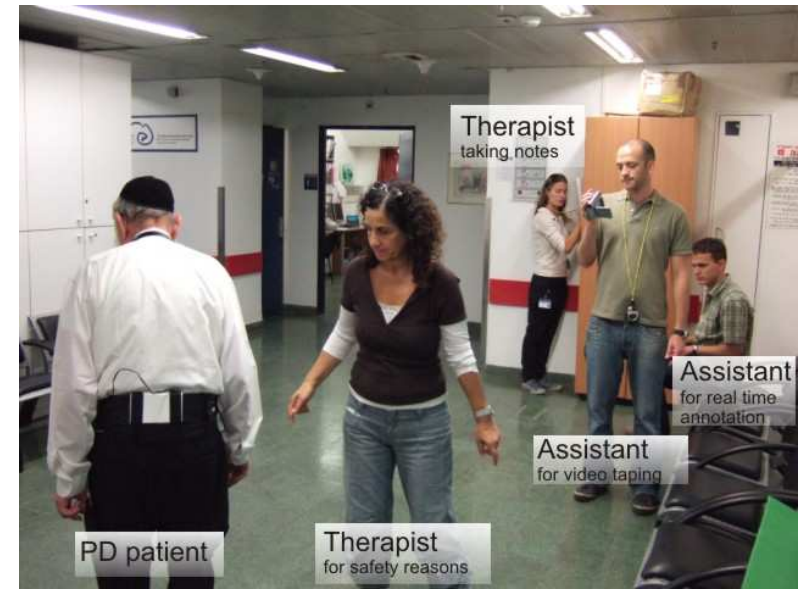

Figure 2: Snapshot of the study, depicting one PD patient, the therapist (near the patient for safety reasons) and the research assistants (more remotely from the patient) who were documenting the trials.

with several turns, the third task included more typical activities of daily living at home as for example moving around rooms, going to the kitchen, carrying a glass of water. Participants walked without assistance, but with a therapist close by for safety reasons (see figure 2), at their own natural pace. A detailed description of the protocol is given in $[1]$.

Real-time annotation and simultaneous video taping were used to determine the number and exact times of FOG episodes (figure 2). Each patient was watched closely by an assistant who real-time annotated the patients' current activity (standing, walking, turning and freezing). In a subsequent process professional physiotherapists analyzed the video recordings to identify the FOG events and determine the exact start times, durations and end times.

\section{RESULTS AND DISCUSSION}

Eight patients out of the ten exhibited FOG during the study, two patients did not have any freeze event (patient 04 and 10). The walking distance and number of turns depended on the patients' gait speed. One patient could not perform the ADL part (patient 08). Overall $8 \mathrm{~h} \mathrm{20min} \mathrm{of}$ data have been recorded. 237 FOG events (range 0-66 per

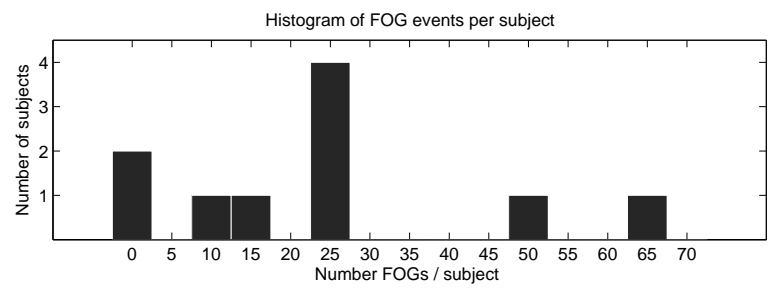

Figure 3: Number of FOG events per patient.

patient; mean 23.7 [S.D. 20.7]) have been identified from the video recordings by the physiotherapists. The detailed 
distribution of number of FOG per patients is given in figure 3. The length of the FOG events ranged from $0.5 \mathrm{sec}$ to $40.5 \mathrm{sec}$ (mean $7.3 \mathrm{sec}$ [S.D. $46.7 \mathrm{sec}$ ]). $50 \%$ of the FOG episodes lasted less than $5.4 \mathrm{sec}$, and the majority of $93.2 \%$ of FOG events had a duration less than 20 sec. The detailed distribution of number of FOG events within $1 \mathrm{sec}$ bins is given in figure 4 . We did not experience any technical prob-

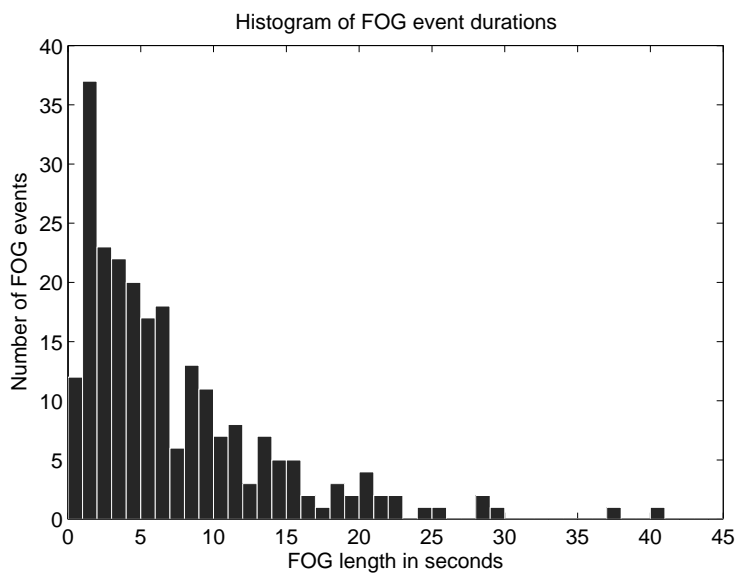

Figure 4: Distribution of duration of FOG events.

lems during the recordings. For the online detection of FOG we used the vertical axis of the sensor at the ankle and one global parameter set. The auditory cueing started properly whenever a FOG episode was detected.

\subsection{Algorithm performance evaluation}

The algorithm detection performance is evaluated using a $0.5 \mathrm{sec}$ based frame evaluation, allowing a maximum detection delay of $2 \mathrm{sec}$ (tolerance). The reference for all our evaluations is the video annotation of the physiotherapists. Figure 5 shows examples of the detection evaluation. The evaluation is done on a time basis, because we have to account for the duration of the freezes and freeze detection, and not just their occurrence as one single event. Each $0.5 \mathrm{sec}$ frame is compared with the reference annotation. 'True positive' (TP) are all frames counted where the algorithm has correctly detected FOG. At the beginning and end of each FOG episode there is a tolerance of $2 \mathrm{sec}$ (see figure 5c). 'False positive' (FP) are all frames which have been detected by the algorithm as being FOG, however there is no FOG (see figure 5b). 'False negative' (FN) are all frames counted where the algorithm has missed to detect FOG (see figure $5 \mathrm{a}+\mathrm{d})$. Finally all frames where correctly no FOG is detected are counted as 'true positive' (TN).

The Sensitivity $\left(\right.$ Sens $\left.=\frac{T P}{T P+F N}\right)$ measures the proportion of correct detected 'FOG time' to whole 'FOG time'. The Specificity $\left(S p e c=\frac{T N}{T N+F P}\right)$ measures the proportion of correctly as 'no FOG' detected time to whole time where no FOG occurred.

$96.2 \%$ of the identified FOG episodes $(n=237)$ were detected online by the wearable device. The frame based sensitivity and specificity of the online detected FOG were $73.1 \%$ and $81.6 \%$, respectively. The evaluation is based on $0.5 \mathrm{sec}$ frames with a 2 sec delay tolerance.
Figure 6 depicts the detection accuracy in terms of sensitivity and specificity for each patient individually. It can be seen that the system did not work equally well on all patients. Worst results in terms of specificity performance was obtained for patient 01 . Only $38.7 \%$ specificity was achieved (with $97.1 \%$ sensitivity). On the other hand worst results in terms of sensitivity was obtained for patient 08 with a sensitivity of $28.7 \%$ (specificity of $87.7 \%$ ). We identified these large variations to be caused by the different walking styles of the patients. For example, patient 01 suffered from foot drop while walking, which we call a saccadic walking style. For patient 01 the system was most of the time not able to distinguish between walking periods and very short freezing events using the global algorithm parameter settings.
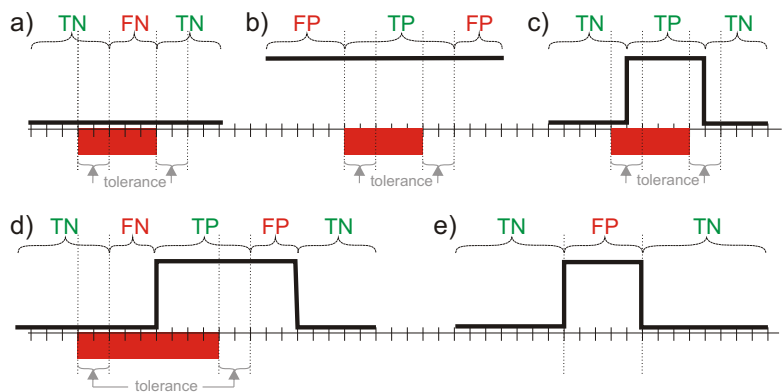

Figure 5: Examples of the FOG detection performance evaluation. The red bars indicate the reference annotation of FOG. The black lines are the FOG detection signal ( 0 = 'no FOG'; $1=$ FOG). Vertical dashed lines mark the allowed tolerance. a) Nothing detected: Sens $=100 \%$, Spec $=0 \%$ b) Everything 'detected': Sens $=0 \%$, Spec $=100 \%$ c) Correct detected within the tolerance: Sens = $100 \%$, Spec $=100 \%$ d) Correct detected but with too big offset: Sens $=66.7 \%$, Spec $=78.6 \% \mathrm{~d}$ ) Wrong detected event.

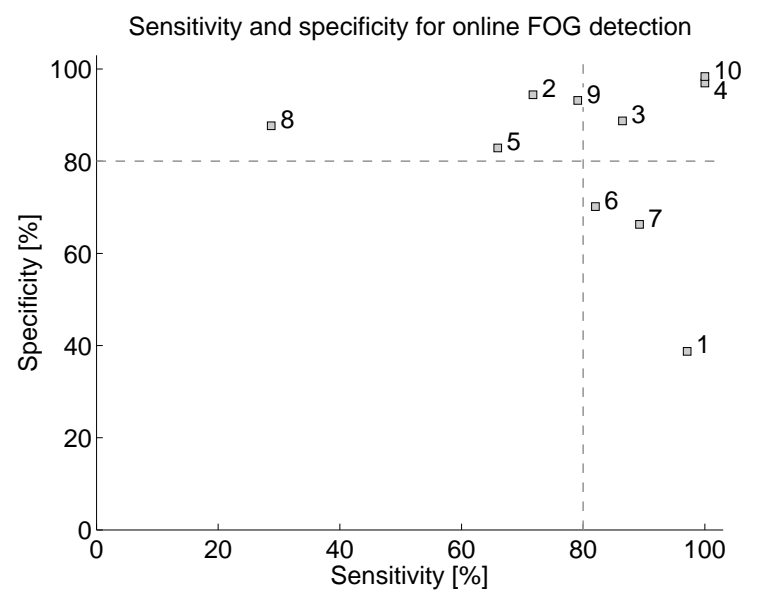

Figure 6: Sensitivity and specificity distribution for the online detection accuracy. Numbers correspond to patient ID. 


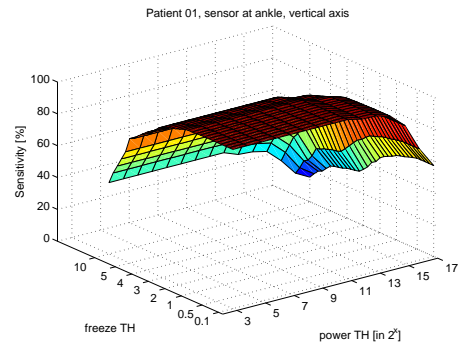

(a) Sensitivity

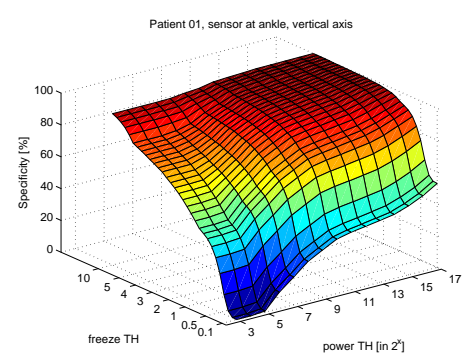

(b) Specificity

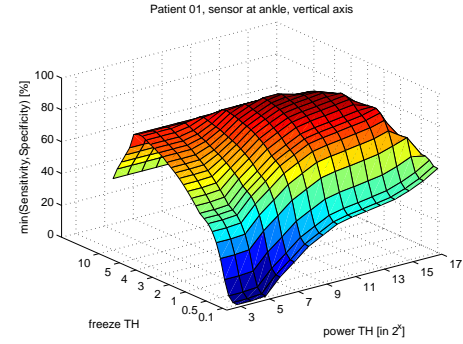

(c) $\operatorname{Min}($ Sens,Spec)

Figure 7: Sensitivity, Specificity and Min(Sens,Spec) plots for patient 01 (vertical axis of sensor at ankle).

\subsection{Algorithm complexity analysis}

In this study we used our modular research platform for a first investigation of algorithm performance and complexity. However our end-goal is to integrate sensing and processing into a single miniature sensor node which can run for several days. Therefore limiting the complexity of the algorithm is important.

Our analysis of the algorithm complexity has shown that the whole computation can be done with 353 multiplications, 449 addition, 2 comparisons and some bit shifts every 0.5 sec. These numbers are compose of:

- $\frac{N_{F F T}}{2} \cdot \log _{2}\left(N_{F F T}\right)$ complex multiplies and $N_{F F T}$. $\log _{2}\left(N_{F F T}\right)$ complex additions (for $N_{F F T}$ a power of 2) to compute the FFT using the radix-2 Cooley-Tukey FFT algorithm. One complex multiplication are 3 real multiplications and one bit shift (multiplication by 2). One complex addition are 2 real additions. Because our input data are purely real, the outputs satisfy the symmetry $X_{N-k}=X_{k}^{*}$ and roughly a factor of two in time and memory can be saved by removing the redundant parts of the computation. Minimal $f_{S R}$ needed is $16 \mathrm{~Hz}$, therefore minimal $N_{F F T}=64$.

- $2 \cdot\left(\mathrm{x}_{L B}+\mathrm{x}_{F B}\right)$ real multiplications and $\mathrm{x}_{L B}+\mathrm{x}_{F B}$ real additions to compute the power spectrum of the relevant frequency bands with $\mathrm{x}_{L B}=(3 \mathrm{~Hz}-0.5 \mathrm{~Hz}) / f_{\text {res }}+$ 1 number of operations in the 'Loco band' and $\mathrm{x}_{F B}=$ $(3 \mathrm{~Hz}-0.5 \mathrm{~Hz}) / f_{\text {res }}+1$ number of operations in the 'Freeze band'.

- $\mathrm{x}_{L B}+\mathrm{x}_{F B}+1$ additions to sum up the power spectrum of the 'Loco band', 'Freeze band' and 'Overall sum'.

- One division, whereas a division by a number is a multiplication by its reciprocal.

- Two comparisons with the thresholds.

\subsection{Characterization of parameter influence}

We have analyzed the influence of the two threshold parameters PowerTH and FreezeTH on the algorithm detection performance. All analysis in this subsection are done on the sensor data of the ankle position, vertical axis, which seems on a first guess to be the best position. Figure 7 depicts the performance evaluation for different parameter settings for patient 01 . In figure $7 \mathrm{a}$ ) the sensitivity of the algorithm for the different parameter combinations is plotted, respectively figure $7 \mathrm{~b}$ ) depicts the specificity. As expected there is a trade-off between sensitivity and specificity - highest sensitivity is achieved with minimal threshold at the expense of a lower specificity. On the other hand highest specificity is achieved for high thresholds at the expense of lower sensitivity. There are different possibilities to decide for the best combination of sensitivity and specificity. For our work we have chosen to take the minimum between sensitivity and specificity plotted as $\min ($ Sens,Spec) in figure $7 \mathrm{c})$.

The maximum in the $\min (S e n s, S p e c)$-plain corresponds to the minimum equal error rate (EER) and is the 'optimal' point. In figure 7 one can see that by adapting properly the two threshold parameter of the algorithm, results of $95.9 \%$ sensitivtiy with $92.7 \%$ specificity could be achieved for patient 01 . The performance of the algorithm on the data of patient 01 is relative insensitive to small variations of the FreezeTH and the PowerTH.

In figure 8 the $\min (\mathrm{Sens}, \mathrm{Spec}$ )-plain for patient 02 is depicted. One can see that the performance on this data is much more sensitive on the FreezeTH, however almost insensitive over a very broad range of the PowerTH $\left(2^{7}-2^{15}\right)$. Figure 9 depicts the distribution of the optimal parameter for each individual patient (patient 04 and 10 are excluded because they did not have FOG). The black dots mark the optimal parameter combination. The gray areas mark all parameter combinations where the detection accuracy is less than $5 \%$ lower than the maximum possible. One can see the link between the distribution of the optimal parameter shown in figure 9 and the $\min (S e n s, S p e c)$-plain when comparing the 'max $5 \%$ distribution' of patient 01 in figure 9 with figure $7 \mathrm{c}$ ) or the 'max $5 \%$ distribution' of patient 02 in figure 9 with figure 8 .

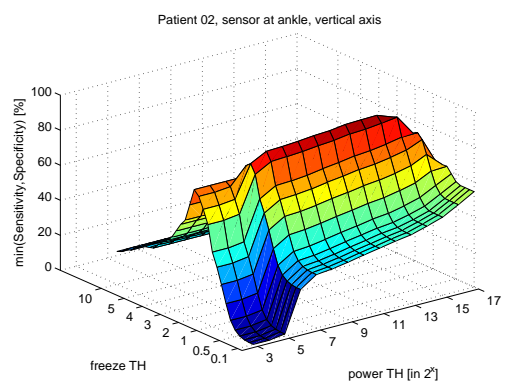

Figure 8: Min(Sens,Spec)-plot for patient 02. 
We are interested in comparing a global optimization of the threshold parameters against a user-specific optimization of the parameters. By optimizing the parameters for each user individually, we can compute the theoretical maximum performance, achievable with the given algorithm, assuming optimal individual training of the algorithm parameter. The optimal sensitivity and specificity we can expect with an optimal user specific parameter set is depicted in figure 11a). This is a theoretical result we use as reference to compare how much we can gain by a user specific training. Potential improvement of user specific training is reported by measuring the difference between Sens opt $_{\text {- Sens }}$ current and Spec $_{\text {opt }}$ - Spec current.

Performance with global parameters is always computed using a leave-one-out cross-validation, meaning that the global parameter is trained on $N-1$ subjects and performance is tested on the remaining subject. The results with global parameters is plotted in figure 11b). The algorithm performance with global parameters is in average 11.1\% (STD $\pm 5.3 \%$ ) away from the optimal user specific performance.

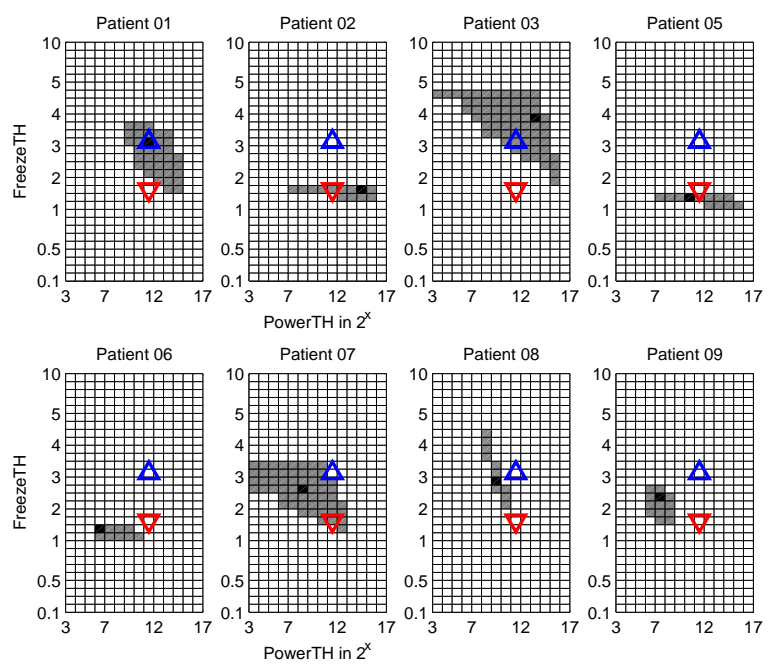

Figure 9: Distribution of optimal parameter. The black dots mark the optimal parameter combination. The gray areas mark the parameter combinations for the optimal $5 \%$ performance. Upward and downward pointing triangles mark the group parameter sets for smooth and saccadic walking styles.

In the plots of figure 8 we identified two main groups:

i) patients with an optimal FreezeTH around 1.5;

ii) patients with an optimal FreezeTH around 3.

Based on these findings we analyzed the detection performance when grouping the 10 patients into the two groups. The FreezeTH and PowerTH for the two groups are chosen manually as visual average of the observation (no special algorithmic training on the data). The first group consists of patients with a more smooth walking style $\left(\right.$ Freeze $T H_{\text {low }}=$ $1.5)$ and the second group consisting of patients with a more saccadic walking style $\left(\right.$ FreezeTH $H_{\text {high }}=2 \cdot$ FreezeTH $\left.T H_{\text {low }}\right)$. The two parameter sets are marked in the plots of figure 9 by an upward and a downward pointing triangle. Group membership is done according to the smaller distance between the optimal user specific parameter to the group parameter.
Figure 11c) presents the sensitivity/specificity with parameters optimized for the smooth and saccadic walkers. The performance is in average $3.7 \%$ (STD $\pm 2.8 \%$ ) away from the optimal user specific performance.

In the plots of figure 8 we also see that the algorithm performance for patient 08 is very PowerTH sensitive. This is not the case for the other patients. Patient 08 is the patient most effected by the PD (H\&Y of 4) and with the most difficulties to walk. Most of the time she had akinesic ('without motion') FOG, not distinguishable from voluntary standing with acceleration (motion) sensors. This is an explanation for the PowerTH sensitivity. Patient 08 is also the patient with the lowest sensitivity of $76.1 \%$ and specificity of $78.1 \%$ for the optimal user specific parameter set.

\subsection{Sensor placement characterization}

We investigate the performance of the system using other sensor placement and measurement axis to understand which is the best location in terms of the trade-off between wearability and performance.

Figure 10 depicts a 4 min signal extract of patient 02 of the sensor at the hip and at the ankle. Clearly there is a dif-
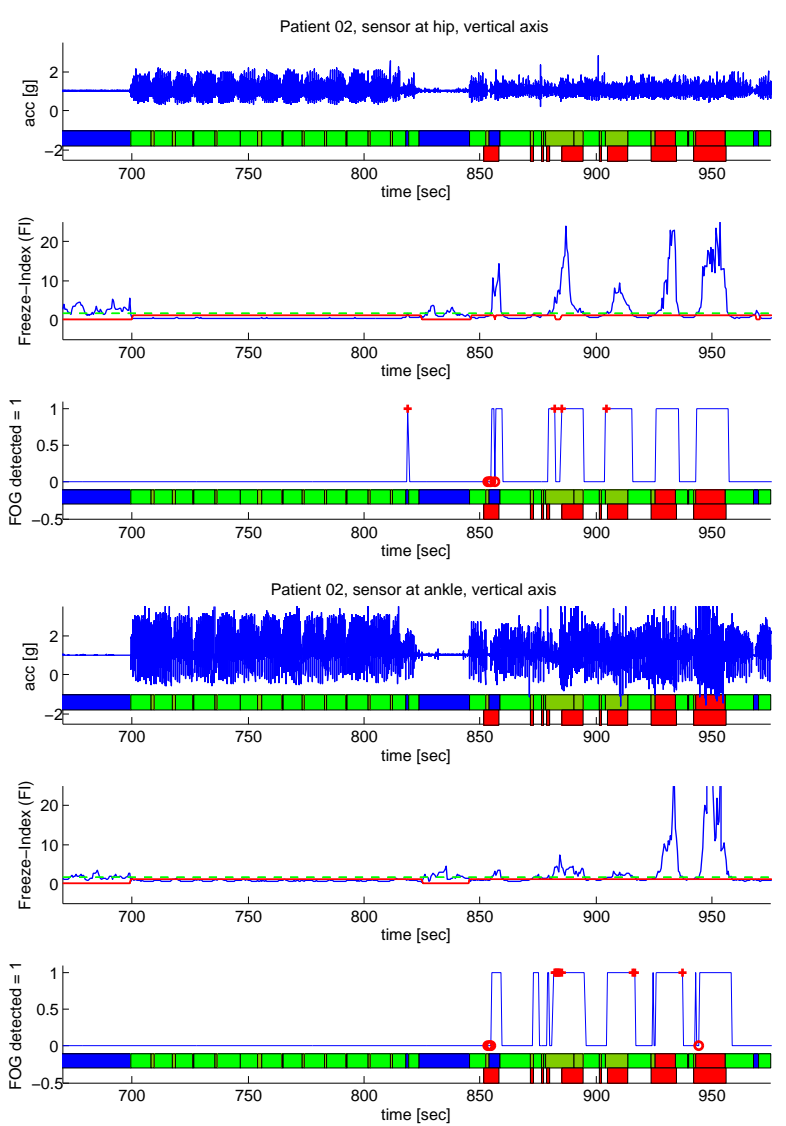

Figure 10: A 4 min signal extract from patient 02 of the sensor data at the hip (upper plot) and at the ankle (lower plot) together with the freeze index (FI) and the FOG detected parts. 


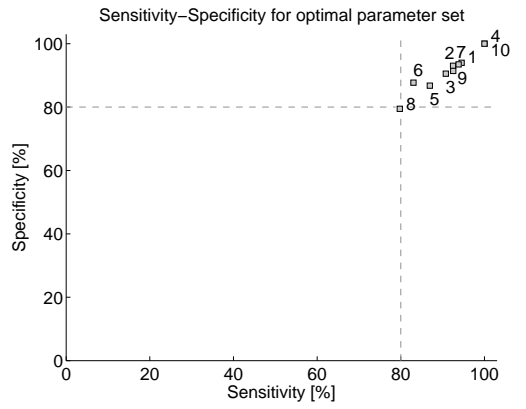

(a) Sens-Spec for optimal patient dependent parameter.

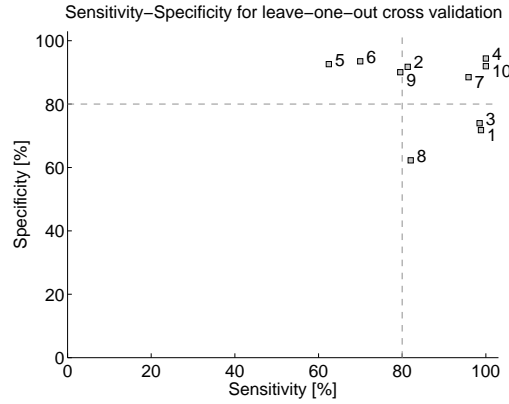

(b) Sens-Spec for leave-one-out cross validation.

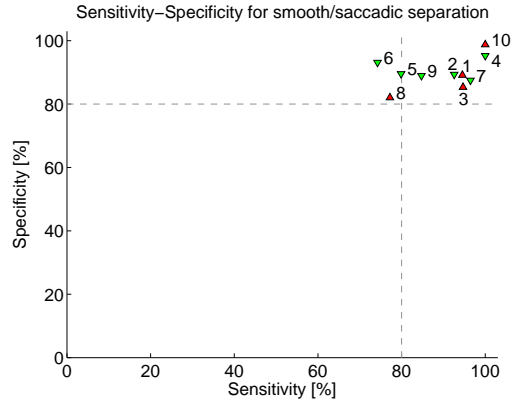

(c) Sens-Spec for smooth and saccadic walking parameter set.

Figure 11: Sensitivity and specificity plots for different parameter sets evaluated on data of the vertical axis of the ankle sensor.

ference between both signals - the signal at the hip is much smaller (damped), but the motion is still very well visible. In the FI plot we see that the attenuation has no big influence on the power spectrum and therefore on the algorithm performance. In some cases, as in this example, the FI derived from the sensor data at the hip is even better than the FI derived from the sensor data at the ankle. Short FOG events however are more likely to be missed.

Table 1 lists the performance of the algorithm using the leave-one-out cross-validation for the 12 possible combinations of the three sensor positions (ankle, knee, hip) and the three possible axis ( $x=$ horizontal forward, $y=$ vertical, $z=$ horizontal lateral) plus the magnitude of all three axis $\left(n=\sqrt{x^{2}+y^{2}+z^{2}}\right)$.

Best results are achieved using the vertical axis of the sensor at the knee. But placing the sensor on the thigh just above the knee is the most inconvenient position to wear [1]. However the detection accuracy at the other positions is still

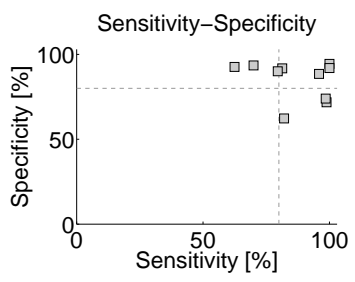

(a) Ankle

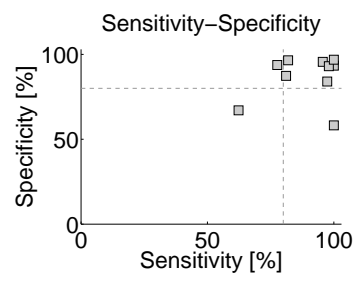

(b) Knee

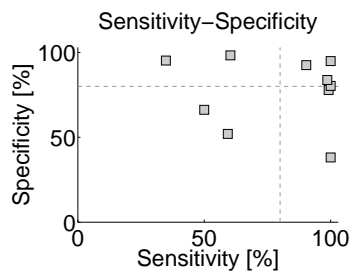

(c) Hip

Figure 12: Sensitivity and specificity distribution for a global threshold set when placing the sensor at different positions. quite good. These results are very promising, because the sensors can be placed at a more convenient body position without loosing much on the accuracy.

The STD results in table 1 indicate for which location a parameter training based on data of other patients is more difficult. A high STD is an indication for being more sensitive on the correct parameter set than a low STD. The variation can also be seen when plotting the results as depicted in figure 12 for the three sensor locations alway using the vertical axis.

\section{CONCLUSION}

To our knowledge, this is the first time that FOG has been automatically detected online by a wearable device. The system detected the FOG events with a sensitivity of $73.1 \%$ and a specifity of $81.6 \%$.

Given the large inter patient variation in the gait performance of the PD patients, we have shown that at least a rough segmentation of smooth and saccadic walking together with specific sensitivity adjustment can improve the detection performance to $85.9 \%$ sensitivity and $90.9 \%$ specificity. With a global threshold (the leave-one-out cross validation) a detection accuracy of $87.1 \%$ sensitivity and $86.9 \%$ specificity was achieved.

The analysis of three different sensor locations has shown that all three locations could be use for FOG detection with minor differences in detection performance. The ankle position is especially interesting for the integration of the sensor into a shoe. At the hip position the sensor could be integrated into a belt. However the sensor location at the hip is much more parameter sensitive and therefore less preferable for real world application. Performance may be further increased by sensor fusion, especially for those patients where freezing does not translate in tremors in both legs.

For our first investigations we have used our flexible but bulky general purpose wearable computing platform. The algorithmic complexity however allows for a specialized system designed for this task that is miniaturized to a size of a button with the FOG algorithms included in the sensor node itself. Roggen et al. have shown that computations similar in complexity to those carried out in this work are suited for online implementation in miniature sensor nodes of the size of a button [11]. Such a system could be entirely integrated 


\begin{tabular}{|c|c|c|c|c|c|c|c|c|}
\hline Sensor at ankle & \multicolumn{2}{|c|}{ horizontal forward $[x]$} & \multicolumn{2}{|c|}{ vertical $[y]$} & \multicolumn{2}{|c|}{ horizontal lateral $[z]$} & \multicolumn{2}{|c|}{ magnitude $[n]$} \\
\hline Sensitivity & $87.15 \%$ & $\pm 16.11 \%$ & $81.46 \%$ & $\pm 13.72 \%$ & $80.20 \%$ & $\pm 13.24 \%$ & $79.23 \%$ & $\pm 14.67 \%$ \\
\hline Specificity & $86.60 \%$ & $\pm 14.49 \%$ & $86.90 \%$ & $\pm 11.37 \%$ & $80.79 \%$ & $\pm 19.33 \%$ & $86.33 \%$ & $\pm 8.92 \%$ \\
\hline \multicolumn{9}{|l|}{ Sensor at knee } \\
\hline Sensitivity & $75.81 \%$ & $\pm 20.21 \%$ & $85.33 \%$ & $\pm 12.96 \%$ & $81.85 \%$ & $\pm 18.22 \%$ & $82.43 \%$ & $\pm 15.40 \%$ \\
\hline Specificity & $84.75 \%$ & $\pm 15.91 \%$ & $87.84 \%$ & $\pm 13.42 \%$ & $84.06 \%$ & $\pm 19.77 \%$ & $83.30 \%$ & $\pm 13.29 \%$ \\
\hline \multicolumn{9}{|l|}{ Sensor at hip } \\
\hline Sensitivity & $81.15 \%$ & $\pm 19.00 \%$ & $70.59 \%$ & $\pm 25.33 \%$ & $77.76 \%$ & $\pm 31.75 \%$ & $77.55 \%$ & $\pm 18.94 \%$ \\
\hline Specificity & $83.57 \%$ & $\pm 28.13 \%$ & $79.37 \%$ & $\pm 20.12 \%$ & $79.32 \%$ & $\pm 21.97 \%$ & $80.38 \%$ & $\pm 23.69 \%$ \\
\hline
\end{tabular}

Table 1: Sensor position evaluation: Sensitivity and specificity ( \pm std) for each combination of sensor position (ankle, knee, hip) and axis orientation.

into (or attached to) normal shoes of the patient, and only the trigger for the external cueing signal is transmitted to the feedback device. The external cueing signal can be given by a hearing aid like device or even the hearing aid itself.

\section{Acknowledgment}

This paper describes work carried out in the context of the FP6 project DAPHNet, FET-018474-2. We thank the patients for their participation, time and effort, and Ms. Inbal Meidan, Ms Noit Inbar, Ms Talia Herman, Ms Marina Brozgol and Mr. Eliya Shaviv for invaluable assistance.

\section{REFERENCES}

[1] M. Bächlin, M. Plotnik, D. Roggen, N. Inbar, I. Meidan, J. Hausdorff, and G. Tröster. Parkinson patients' perspective on context aware wearable technology for auditive assistance. In Proc. of the 3rd Int. Conf. on Pervasive Computing Technologies for Healthcare, 2009.

[2] M. Bächlin, D. Roggen, and G. Tröster. Context-aware platform for long-term life style management and medical signal analysis. In In Proceeding of the 2nd SENSATION International Conference, Chania, Greece, June 2007.

[3] D. Bannach, K. Kunze, P. Lukowicz, and O. Amft. Distributed modular toolbox for multi-modal context recognition. In ARCS 2006: Proceedings of the 19th International Conference on Architecture of Computing Systems., pages 99-113, March 2006.

[4] B. R. Bloem, J. M. Hausdorff, J. E. Visser, and N. Giladi. Falls and freezing of gait in parkinsonŠs disease: A review of two interconnected, episodic phenomena. Movement Disorders, 19(8):871-884, 2008.

[5] H. Braak, E. Ghebremedhin, U. Rüb, H. Bratzke, and K. D. Tredici. Stages in the development of parkinson's disease-related pathology. Cell and Tissue Research, 318(1):121-134, October 2004.

[6] A. G. de Boer, W. Wijker, J. D. Speelman, and J. C. de Haes. Quality of life in patients with parkinson's disease: development of a questionnaire. Journal of Neurology, Neurosurgery and Psychiatry, 61(1):70- 74, July 1996.
[7] J. Jankovic. Parkinson's disease: clinical features and diagnosis. J Neurol Neurosurg Psychiatry, 79(4):368-376, 2008.

[8] I. Lim, E. van Wegen, C. de Goede, M. Deutekom, A. Nieuwboer, A. Willems, D. Jones, L. Rochester, and G. Kwakkel. Effects of external rhythmical cueing on gait in patients with parkinson's disease: a systematic review. Clinical Rehabilitation, 19(7):695-713, 2005.

[9] M. Macht, Y. Kaussner, J. C. Möller, K. Stiasny-Kolster, K. M. Eggert, H.-P. Krüger, and H. Ellgring. Predictors of freezing in parkinson's disease: A survey of 6,620 patients. Movement Disorders, 22(7):953-956, 2007.

[10] S. T. Moore, H. G. MacDougall, and W. G. Ondo. Ambulatory monitoring of freezing of gait in parkinson's disease. Journal of Neuroscience Methods, 167(2):340 - 348, 2008.

[11] D. Roggen, N. B. Bharatula, M. Stäger, P. Lukowicz, and G. Tröster. From sensors to miniature networked sensorbuttons. In In Proc. of the 3rd Int. Conf. on Networked Sensing Systems (INSS06), pages 119-122, 2006.

[12] J. K. Sheffield and J. Jankovic. Botulinum toxin in the treatment of tremors, dystonias, sialorrhea and other symptoms associated with parkinson's disease. Expert Review of Neurotherapeutics, 7(6):637-647, June 2007.

[13] M. H. Thaut, G. C. McIntosh, R. R. Rice, R. A. Miller, J. Rathbun, and J. M. Brault. Rhythmic auditory stimulation in gait training for parkinsonŠs disease patients. Movement Disorders, 11(2):193-200, 1996.

[14] E. van Wegen, C. de Goede, I. Lim, M. Rietberg, A. Nieuwboer, A. Willems, D. Jones, L. Rochester, V. Hetherington, H. Berendse, J. Zijlmans, E. Wolters, and G. Kwakkel. The effect of rhythmic somatosensory cueing on gait in patients with parkinson's disease. Journal of the Neurological Sciences, 248(1-2):210 - 214, 2006. 